\title{
Exploring the energy landscape
}

\author{
The search for emerging properties in far-from-equilibrium supramolecular systems is just beginning.
}

In a Review on supramolecular systems chemistry (page 111) Elio Mattia and Sijbren Otto - who are based at the Institute of University of Groningen guide us through the major conceptual developments in the field of supramolecular chemistry, a field that has been described ${ }^{1}$ as 'chemistry beyond the molecule'. Supramolecular chemistry deals with weak, reversible molecular interactions that are harnessed to make large aggregates and the approach is a fundamental component of self-assembly techniques and bottom-up fabrication processes. Mattia and Otto first look at the initial approach to supramolecular chemistry, which has principally involved thermodynamic equilibrium assemblies, and then move on to more recent developments that exploit states in a kinetic trap (a local minimum with potential energy barriers high enough to trap a specific configuration). But perhaps the most exciting aspect of the Review comes from the discussion of far-from-equilibrium systems. These are chemical systems that cannot be defined by a single supramolecular structure; rather, their properties come from a continuous interchange among a series of different structures as the system moves along a potential energy landscape. To remain far from equilibrium, these supramolecular systems require a constant supply of energy that is then dissipated into the environment.

Alongside the supramolecular chemistry of far-from-equilibrium systems, Mattia and Otto highlight how the topic ties in with the field of systems chemistry, the ultimate goal of which is to understand the chemical basis of life ${ }^{2}$. Systems chemists look for new chemical properties that emerge from the interaction between chemical systems. And because far-fromequilibrium supramolecular chemistry studies the interplay between different states and how these interact with each other and the environment, the confluence between the two fields seems natural. Published examples of emerging properties that are sustained by a continuous supply of energy include the formation of compartments, concentration gradients and directional motion of molecular components ${ }^{3}$. If harnessed in a concerted fashion, directed molecular motion can be made to do work against the environment. An example of this is reported by Nicolas Giuseppone and colleagues at the University of Strasburg on page 161. In particular, they show that thousands of molecular rotors attached to a polymer network can work in unison under light irradiation to produce a macroscopic structural modification of a gel.

Although in the long run the fields of supramolecular chemistry and systems chemistry may provide a new angle in our understanding of life from a chemical perspective, it is not unreasonable that the collaboration between researchers in the two fields will also be useful for designing new materials that adapt, respond and exhibit different functionalities as the environmental conditions change.

\footnotetext{
References

1. Lehn, J.-M. Angew Chem. Int. Ed. 27, 89-112 (1988).

2. Pross, A. What is Life? How Chemistry Becomes Biology (Oxford Univ. Press, 2012).

3. Ruiz-Mirazo, K., Briones, C. \& de la Escosura, A. Chem. Rev. 114, 285-366 (2014).
}

\section{Can we be more social?}

\section{The social and economic issues surrounding nanotechnology should not be forgotten.}

\begin{abstract}
The principal aim of Nature Nanotechnology is to publish the most significant research in all areas of nanoscience and nanotechnology and, as a result, our authors are a diverse group. They are though made up primarily of physicists, chemists, materials scientists, engineers and biologists. Like all branches of science, nanotechnology also has social and economic implications, and in order to provide a complete picture of the field, these topics should feature in the journal as well.
\end{abstract}

\section{Nanotechnology has social and economic implications, and these topics should feature in the journal as well.}

Since late 2008, we have published social science research that explores the public perceptions of nanotechnology as Letters and Articles, our two primary research formats. (Before this, such work featured only in our Commentary section.) However, we have published only a handful of research papers on the topic.

To date, our coverage of the social and economic implications of nanotechnology has occurred primarily through the 'front half' of the journal. For example, our Thesis writers, and Chris Toumey in particular, often discuss historical, philosophical and social themes. Alternatively, we have published Commentaries on economic issues such as the insurability of nanotechnology and the development of the nanobiotechnology industry. Furthermore, we have published a focus issue on nanoscience education, which subsequently led to the launch of a regular page dedicated to education called In the Classroom. This page has featured perspectives from scientists, students and entrepreneurs.

But can we do more? There are certainly areas where we could increase our coverage. For example, papers on policy analysis, economic viability and the scalability of proof-of-principle demonstrations rarely reach our inbox, let alone publication. Such issues, along with work on public perception, are essential components in the development of nanotechnology. To deliver the complete picture of the field we strive for at the journal, we must also aim to publish significant opinions, analysis and original research in all of these topics. 